\title{
Suppression of Quarkonium Production in Heavy Ion Collisions at RHIC and LHC
}

\author{
L. Gerland $\sharp$, L. Frankfurt $\sharp \ddagger$, M. Strikman $\S$, H. Stöcker $\sharp$, W. \\ Greiner\# \\ \# Institut für Theoretische Physik der J.W.Goethe-Universität \\ Robert-Mayer-Str. 8-10, D-60054 Frankfurt a.M., Germany \\ $\ddagger$ Tel Aviv University, Tel Aviv, Israel \\ $\S$ Department of Physics, Pennsylvania State University \\ University Park, PA 16802, USA
}

\begin{abstract}
A model for the production of quarkonium states in the midrapidity region at RHIC and LHC energy range is presented which explores well understood properties of QCD only. An increase of the quarkonium hadronisation time with the initial energy leads to a gradual change of the most important phenomena from fixed target- to collider-energies. We evaluate nuclear effects in the quarkonium production due to medium modification of the momentum distribution of the heavy quarks produced in the hard interactions, i.e. due to the broadening of the transverse momentum distribution. Other nuclear effects, i.e. nuclear shadowing and parton energy loss, are also evaluated.
\end{abstract}

\section{Introduction}

At fixed target energies the hadronisation time $t_{f}$ for $Q \bar{Q}$ pair is small as compared to the nuclear radii, at collider energies this time is much larger than the size of the nucleus. Thus, at fixed target energies, the interaction of heavy quarkonium with the surrounding medium can be described effectively as hadronic [1], 2] even within the hadronisation time. However, at collider energies a description of the process in terms of hadron degrees of freedom becomes questionable since a bound state of the heavy quark pair is formed far outside of the nucleus.

One major effect at collider energies is the transverse momentum broadening of $Q \bar{Q}$ pairs travelling through a medium. This changes the overlap integral between the $Q \bar{Q}$ pair and the wave functions of the heavy quarkonia. It is important that soft interactions of heavy quarks with nucleons do not change the longitudinal momentum of heavy quarks which may appear sensitive to the interaction with comovers which are neglected in this paper. Nuclear effects in the momentum distribution within the heavy quark pair due to the radiation of gluons which is sensitive to the properties of the QCD-matter produced in a nucleus-nucleus collision at collider energies [3] will be estimated separately in the section six.

The typical time scales for the creation of a hidden heavy flavour state are investigated in section two, in the third section a model is developed to calculate the influence of nuclear effects on the production of states with hidden heavy flavor in the midrapidity region at collider energies. In the fourth section this model will be used to predict heavy quarkonium products in $A B$ collisions at RHIC and LHC and 
in section five and six quarkonium suppression due to nuclear effects on the parton distribution functions and parton energy loss are evaluated.

\section{Coherence Lengths}

There are different time scales relevant for the production of quarkonium states:

1) the time needed to produce a heavy quark pair in a hard collision,

2 ) the time needed for a $Q \bar{Q}$ pair to form a bound state.

The production time of a $Q \bar{Q}$ pair in its rest frame is given by $\tau_{p}=\frac{1}{m_{Q}}$. This is $0.13 \mathrm{fm} / \mathrm{c}$ for $c \bar{c}$ and $0.05 \mathrm{fm} / \mathrm{c}$ for $b \bar{b}$. The Lorentz factor of the pair at midrapidity in the rest frame of the target is $\gamma=\frac{\sqrt{s}}{2 m_{N}} \approx 10,20,100$ and 3000 for a Quarkonium state in the midrapidity region at SPS, Fermilab, RHIC and LHC energies. Thus, at SPS and Fermilab fixed target energies $\gamma c \tau_{p}$ is smaller than the average internucleon distance in nuclei $r_{N N} \approx 1.8 \mathrm{fm}$. Thus, the production of heavy quark pairs is incoherent. However, at RHIC the production distance of $c \bar{c}$ pairs is already as large as the diameter of a gold nucleus, and for $b \bar{b}$ pairs $c \tau_{p}>1.8 \mathrm{fm}$, but this is still small as compared to the nuclear radius. At LHC both production distances exceed the diameter of a lead nucleus by an order of magnitude.

The hadronisation time $t_{H}$ resp. the coherence length $l_{c}$ of heavy Quarkonium is $l_{c}=c \cdot t_{H}=\frac{1}{\Delta E} \approx \frac{\gamma}{\Delta M}$ with:

$\Delta E=\sqrt{p^{2}+\left(M_{Q \bar{Q}}+\Delta M\right)^{2}}-\sqrt{p^{2}+M_{Q \bar{Q}}^{2}} \approx \frac{\left(M_{Q \bar{Q}}+\Delta M\right)^{2}-M_{Q \bar{Q}}^{2}}{2 p} \approx \frac{M_{Q \bar{Q}} \Delta M}{p}=\frac{\Delta M}{\gamma}$. Here $p$ is the momentum of the Quarkonium in the rest frame of the target, $M_{Q \bar{Q}}$ is the mass of the $Q \bar{Q}$-pair and $\Delta M=\int \psi^{2}(k) \frac{k^{2}}{M_{Q}} \mathrm{~d}^{3} k / \int \psi^{2}(k) \mathrm{d}^{3} k$, where $\psi(k)$ is the wavefunction of the Quarkonium state in momentum space. That means $\Delta M$ is the average kinetic energy of the $Q \bar{Q}$-pair in the bound state and $l_{c} / \gamma=0.44(0.34)$ fm for the $J / \Psi(\Upsilon)$.

Thus, for charm and bottom production at RHIC and LHC $l_{c}>2 \cdot R_{A}\left(R_{A}\right.$ is the nuclear radius), $l_{c}<2 \cdot R_{A}$ for fixed target at Fermilab $(\gamma \approx 20)$ and at SPS energies. The applicability of the approach developed in this paper requires that $l_{c}>2 \cdot R_{A}$ which is fulfilled at RHIC and LHC (and at large $x_{f}$ at Fermilab fixed target energy range, where $\gamma \sim 100$ also can be reached).

\section{Description of the Model Approach}

We assume here that $Q \bar{Q}$ pairs are produced in $A B$ collisions predominantly in hard collisions. The basic quantity is the cross section of production of $Q \bar{Q}$ pair with light cone momenta $z_{i}, k_{i}$, which we parametrize as $\frac{d \sigma(A B \rightarrow Q \bar{Q}+X)}{\mathrm{d}^{2} k_{1} \mathrm{~d} z_{1} \mathrm{~d} z_{2} \mathrm{~d}^{2} k_{2}}=D_{A B}\left(z_{1}, z_{2}\right)$. $\exp \left(-B(A B)\left(k_{1}^{2}+k_{2}^{2}\right)\right)$. Here $k_{i}\{i=1,2\}$ are the transverse momenta of the $Q$ and the $\bar{Q}$ quark and $z_{i}\{i=1,2\}$ are the fractions of their longitudinal momenta. Such a factorization does not contradict the data in pp collisions [7].

To evaluate the suppression of hidden heavy flavour production resulting from the broadening of the transverse momentum distributions of $Q$ quarks due to final state interaction, we deduce first a relationship between the slopes for the various processes of heavy quark production. In the following we use the relative transverse momentum $k_{t}=\frac{k_{1}-k_{2}}{2}$ and the total transverse momentum $p_{t}=k_{1}+k_{2}$ of the pair, writing $\frac{d \sigma(A B \rightarrow Q \bar{Q}+X)}{\mathrm{d}^{2} k_{t} \mathrm{~d}^{2} p_{t} \mathrm{~d} z_{1} \mathrm{~d} z_{2}}=D_{A B}\left(z_{1}, z_{2}\right) \exp \left(-B(A B)\left(-\frac{p_{t}^{2}}{2}-2 k_{t}^{2}\right)\right)$. 
To take into account possible nuclear effects on the longitudinal momentum distribution we make the ansatz $D_{A B}\left(z_{1}, z_{2}\right)=D(A B) \cdot f_{A B}\left(p_{z}\right) \cdot \exp \left(-\frac{k_{z}^{2}}{C_{A B}^{2}}\right)$. where $p_{z}$ and $k_{z}$ are the total and relative longitudinal momentum. We further assume that $f_{A B}\left(p_{z}\right)=f_{p p}\left(p_{z}\right)$, which means that we neglect parton energy losses of the pair, this effect will be discussed later on. The normalization condition follows from the QCD factorization theorem [4] for the total cross section: $\frac{D(A B)}{B(A B)^{2} \cdot C_{A B}}=\frac{A B \cdot D(p p)}{B(p p)^{2} \cdot C_{p p}}$ The differential cross sections are proportional to the square of the two body wave functions $\phi$.

The production cross section of bound states of heavy quarks is proportional to the overlap integral of the two-body wave function and the wave function of the bound $\psi\left(k_{t}\right)$ state to get $\frac{\mathrm{d}^{3} \sigma(A B \rightarrow \text { Quarkonium }+X)}{\mathrm{d}^{2} p_{t} \mathrm{~d} p_{z}} \propto\left|\left\langle\psi\left(k_{t}, k_{z}\right) \mid \phi_{A B}\left(k_{t}, p_{t}, k_{z}, p_{z}\right)\right\rangle\right|^{2}$. Here we neglected the difference between the current quark mass in the two body wave function and the constituent quark mass in the wave function of the bound state. With this one can evaluate the survival probability: $S \equiv \frac{\sigma(A+B \rightarrow Q \text { uarkonium }+X)}{A B \cdot \sigma(p+p \rightarrow Q \text { uarkonium }+X)}$. Our final result is then

$$
S=\frac{B(A B) C_{A B}}{B(p p) C_{p p}}\left|\frac{\int \mathrm{d}^{3} k \psi(k) \exp \left(-B(A B) k_{t}^{2}\right) \exp \left(-\frac{k_{z}^{2}}{2 C_{A B}^{2}}\right)}{\int \mathrm{d}^{3} k \psi(k) \exp \left(-B(p p) k_{t}^{2}\right) \exp \left(-\frac{k_{z}^{2}}{2 C_{p p}^{2}}\right)}\right|^{2}
$$

up to nuclear effects in the structure function.

Note that if one defines the survival probability as the ratio of the differential cross section $\frac{\mathrm{d}^{2} \sigma}{\mathrm{d}^{2} p_{t}}$ for nuclear and nucleon targets their $p_{t}$ dependence would be a factor $\exp \left(-\frac{B(A B)-B(p p)}{2} p_{t}^{2}\right)$. That means that the $p_{t}$ dependence of $J / \Psi$ suppression is due to the broadening of the transverse momentum distribution as a result of the final sstate interactions of the $Q$ quarks in the nuclear medium.

\section{Predictions for Collider Energies}

To adjust the parameters of eq. (11) we use the quarkonium wave functions of ref. 河, 6] and the following data:

The E789-group (Fermilab) measured a value of $B(p A u)=0.91 \pm 0.12 \mathrm{GeV}^{-2}$ [7] with the fit function $\frac{\mathrm{d} N}{\mathrm{~d} k_{t}^{2}}(D, \bar{D}) \propto \exp \left(-B(p A u) \cdot k_{t}^{2}\right)$ in proton-gold collisions for the transverse momentum distribution of $D$ and $\bar{D}$ mesons. The same group found $n=0.55 \pm 0.01 \mathrm{GeV}^{-2}$ [8] with $\frac{\mathrm{d} N}{\mathrm{~d} p_{t}^{2}}(J / \Psi) \propto \exp \left(-n \cdot p_{t}^{2}\right)$, also in proton-gold for the transverse momentum distribution of $J / \Psi$ 's. Within the error bars these values of $n$ and $B(p A)$ satisfy the relation $n=\frac{B(p A)}{2}$ that was predicted in the previous section. With the fit [9] to the $p_{t}$ broadening of $J / \Psi$ 's this yields $B(p p)=1.18(1.5) \mathrm{GeV}^{-2}$.

There are no data for the nuclear broadening of the longitudinal momentum distribution. We assumed isotropic production in the $c \bar{c}$ rest frame which yields $C_{p p}^{2}=1 /(2 \cdot B(p p)) \approx 0.42 \mathrm{GeV}^{2}$. In fig. 1 the result of eq. (1) is plotted versus the transverse momentum broadening of the $J / \Psi$ 's: $\Delta p_{t}^{2}=2 / B(p p)-2 / B(A B)$. $\Delta p_{t}^{2}=0.48 \mathrm{GeV}^{2}$ was found at Fermilab energies in $p A u . C_{A B}^{2}=C_{p p}^{2}$ is used as a first approximation. $S \approx 1$ for the $J / \Psi$. That means there is practically no change due to the broadening of the transverse momentum distribution.

For the $\Upsilon$ meson we use $\left\langle p_{t}^{2}\right\rangle=2.59 \mathrm{GeV}^{2}$ [10] and thus $B(p p)=0.77 \mathrm{GeV}^{-2}$ and $C_{p p}^{2}=1 /(2 \cdot B(p p)) \approx 0.65 \mathrm{GeV}^{2}$. For the $\Upsilon$ meson production we obtain even a slight enhancement. 


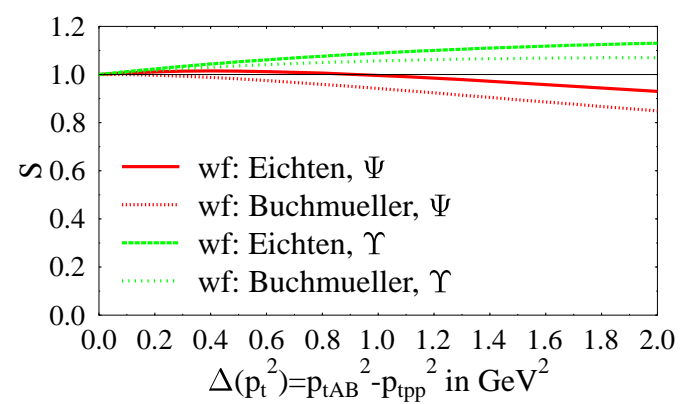

Figure 1: The righthandside of eq.(们) vs. the transverse momentum broadening for the $J / \Psi$ and the $\Upsilon$ is plotted.

Ref. [9 reported that $\Delta p_{t}^{2}$ is nearly a factor 5 larger for $J / \Psi$ 's than for Drell Yan pair production. In our model this is due to a color factor of $\frac{9}{4}$ because $c \bar{c}$ production is dominated by gluon-gluon fusion, while Drell Yan is due to quarkantiquark annihilation and due to the factor of two between the transverse momentum distribution of $c$ resp. $\bar{c}$ Quarks and the bound states evaluated above.

\section{Nuclear Effects on the Gluon Distribution Functions}

The term nuclear effects on the gluon distribution functions means that the ratio of the gluon distribution functions in a nucleus $A$ and a nucleon $N$ differs from 1, i.e $\frac{G_{A}\left(x, Q^{2}\right)}{A G_{N}\left(x, Q^{2}\right)}<1$ in the shadowing region, $\frac{G_{A}\left(x, Q^{2}\right)}{A G_{N}\left(x, Q^{2}\right)}>1$ for $0.1 \leq x \leq 0.3$ and for $x>0.8$. The Bjorken $x$ 's of interest for the production of $J / \Psi$ 's $x=0.0155$ and $\Upsilon$ 's $x=0.0473$ are $x(Q \bar{Q})=\frac{M_{Q \bar{Q}}}{\sqrt{s}}$.

Up to now, the transition points between these regions are not well known. Especially for gluons, the error bars of the data are large. Also from a theoretical point of view they are not unambigious. Therefore, the calculation of these nuclear effects, at not too small $x$, is model dependent. To reduce this model dependence we use here two different recent approaches from ref. [11, 12] (called $E K S$ in the following) and from ref. 13] $(F S)$. (Nuclear shadowing in $(F S)$ has been calculated in a nearly model independent way, but the region of enhancement requires modelling.)

In tab. 1 one can see that quarkonium production at RHIC is enhanced while it is shadowed at LHC. At LHC energies the predictions of both approaches become qualitative different. The latter one based on significantly larger cross section of diffraction in the gluon channel or equivalently on the $9 / 4$ enhancement of color dipole build of color octets. It predicts a stronger shadowing of gluons than the shadowing of quarks and of antiquarks, while in the $E K S$ model it is as strong as the shadowing for sea-quarks.

\section{Suppression due to Parton Energy Loss}

Parton energy loss shifts the partons to smaller x. We use here the parton energy loss model reviewed in ref. [3]. $\Delta E$ is the parton energy loss in a medium. For cold QCDmatter $\Delta E=-4 \mathrm{GeV} \cdot\left(\frac{L}{10 \mathrm{fm}}\right)^{2}$. For hot QCD-matter $\Delta E=-60 \mathrm{GeV} \cdot\left(\frac{L}{10 \mathrm{fm}}\right)^{2}$.

With $L=R_{A u}=7 \mathrm{fm}$ this yields $\Delta E=-2 \mathrm{GeV}$ for cold matter and $\Delta E=-30 \mathrm{GeV}$ for hot matter. For RHIC energies this value has to be divided by 100 since in [3] the parton energy loss was calculated in the rest frame of the target while we are interested in the midrapidity region. Therefore, an initial parton with $x(J / \Psi)=0.017$ in hot QCD-matter is needed to produce a $J / \Psi$. For $p A$ collisions 


\begin{tabular}{|c|c|c|c|c|}
\hline$\sqrt{s}$ in GeV & 60 & 140 & 200 & 6000 \\
\hline$J / \Psi(\mathrm{FS})$ & 1.11 & 1.05 & 1 & 0.65 \\
\hline$J / \Psi(\mathrm{EKS})$ & 1.16 & 1.01 & 0.96 & 0.84 \\
\hline$\Upsilon(\mathrm{FS})$ & 1.09 & 1.04 & 1.09 & 0.83 \\
\hline$\Upsilon(\mathrm{EKS})$ & 1.104 & 1.138 & 1.12 & 0.87 \\
\hline
\end{tabular}

Table 1: $\frac{G_{A}\left(x, Q^{2}\right)}{A G_{N}\left(x, Q^{2}\right)}$ at different initial energies (see text).

this yields an suppression factor of $\frac{G(0.017,3.1 \mathrm{GeV})}{G(0.0155,3.1 \mathrm{GeV})}=0.87$ for the gluon distribution parametrization of Ref. 14, 15. For the $\Upsilon$ this factor is 0.94. For cold QCD-matter this is negligible, since $x=0.0156$ for the $J / \Psi$. This yields a suppression factor of 0.99 . For $A B$ collisions this values have to be squared, since both initial gluons can loose energy. Therefore, the suppression for the $J / \Psi(\Upsilon)$ in hot matter in $A B$ collisions is $0.76(0.88)$. This educated guess must suffice for the time being, because the parton energy loss is theoretical not well understood.

\section{Discussion and Conclusion}

- the relevant physics changes due to coherence length:

$\longrightarrow$ hadronic description at fixed target, partonic at colliders

- nuclear effects on the parton distribution functions yields:

$\longrightarrow$ anti-shadowing at RHIC, shadowing at LHC

- parton energy loss seems to be uneffective in cold QCD matter

- other possible effects not discussed here include:

$\longrightarrow$ interaction with comovers

$\longrightarrow$ long living phase of hot QCD-matter

$\longrightarrow J / \Psi$-production and $\Upsilon$ comes also from the decay of higher resonances

- $p A$ collisions are needed to distinguish between the different effects.

\section{Acknowledgments}

The authors wants to thank V. Guzey for discussions and technical support concerning nuclear effects in the parton distribution functions. LG wants to thank the Josef Buchmann Stiftung. LF acknowledges support by the Alexander von Humboldt Foundation.

\section{References}

[1] Farrar G, Frankfurt L, Strikman M and Li H Phys. Rev. Lett. 64 (1990) 2996

[2] Gerland L, Frankfurt L, Strikman M.Stöcker H and Greiner W Phys. Rev. Lett. 81 (1998) 762

[3] Baier R, Schiff D and Zakharov B G hep-ph/0002198

[4] Bodwin G T Phys. Rev. D31(1985) 2616

[5] Eichten E et al Phys. Rev. D21 (1980), 203

[6] Buchmüller W and Tye S H Phys. Rev. D24 (1981) 132

[7] Leitch M J et al Phys. Rev. Lett. 72 (1994) 2542

[8] M. H. Schub et al Phys. Rev. D52 (1995) 1307

[9] McGaughey P L, Moss J M and Peng J C procs. of NUCOLEX99 and hep-ph/9905447

[10] Alde D M et al Phys. Rev. Lett. 66 (1991) 2285

[11] Eskola K J, Kolhinen V J and Salgado C A Eur. Phys. J. C9 (1999) 61

[12] Eskola K J, Kolhinen V J and Ruuskanen P V Nucl. Phys. B535 (1998) 351

[13] Frankfurt L and Strikman M Eur. Phys. J. A5 (1999)293

[14] H.L. Lai et al Phys. Rev. D55 (1997) 1280

[15] H. L. Lai and W. K. Tung Z. Phys. C74 (1997) 463 